\title{
Performance of Existing Predictor of SubCellular Localization of Long Non-Coding RNA on the New Database
}

\author{
Ying Yuan \\ College of Science \\ Purdue University \\ West Lafayette, USA \\ yuan267@purdue.edu
}

\author{
Bin Yuan \\ School of Electronic Information and \\ Electrical Engineering \\ Shanghai Jiaotong University \\ Shanghai, China \\ yuanbin@sjtu.edu.cn
}

\author{
Hanqiu Mao \\ Department of Software \\ Changshu Kaixi EE Co., LTD \\ Changshu, China \\ maohanqiu@kxeeg.com
}

\begin{abstract}
Long non-coding RNAs (lncRNAs) have important functions in the regulation of life activities at multiple levels, such as chromatin remodeling, transcriptional and posttranscriptional regulation. They have different functions according to the different locations in the cell. Therefore, it is important to obtain the sub-cellular location information to understand the functions of lncRNAs. However, the biochemical experimental way of sub-cellular localization requires a long period and high cost. Currently, many methods of predicting sub-cellular location based on the machine learning and deep learning have been developed to ease the difficulty in detecting the sub-cellular location. We fetched the latest version RNA database to evaluate the performance of existing predictors, lncLocator and iLoc-lncRNA.
\end{abstract}

\section{CCS Concepts}

- General and reference-Cross-computing tools and techniques $\rightarrow$ Evaluation.

\section{Keywords}

Long non-coding RNA; prediction; subcellular localization.

\section{INTRODUCTION}

There are about 20,000 human genomic sequences are determined as protein-coding genes. They account for approximately $2 \%$ of the total human genomic sequences. The other $98 \%$ of the human genome could not be translated into proteins are non-coding RNAs (ncRNAs) [1]. Previously, protein-coding genes were generally thought to be useful genes, while the majority part of the human genomes, nc-RNAs, were considered as "junk". However, recently, many more functions of ncRNAs are discovered from various studies. ncRNAs play important roles in cellular physiological regulation, disease pathological processes, and diagnosis and treatment of human diseases. Those research results on ncRNAs in recent years improve people's understanding of ncRNAs.

Permission to make digital or hard copies of all or part of this work for personal or classroom use is granted without fee provided that copies are not made or distributed for profit or commercial advantage and that copies bear this notice and the full citation on the first page. Copyrights for components of this work owned by others than ACM must be honored. Abstracting with credit is permitted. To copy otherwise, or republish, to post on servers or to redistribute to lists, requires prior specific permission and/or a fee. Request permissions from Permissions@acm.org.

ICCPR 2020, October 30-November 1, 2020, Xiamen, China

(C) 2020 Association for Computing Machinery.

ACM ISBN 978-1-4503-8783-5/20/10 ..\$15.00

https://doi.org/10.1145/3436369.3437447
Long non-coding RNAs (lncRNAs) are a type of RNA with more than 200 nucleotides that do not encode protein [13]. Originally, lncRNAs are considered as byproduct of gene transcription. However, large amount of evidences has shown that lncRNAs are involved in many important regulation functions of gene expression in diseases, including Alzheimer's disease, diabetes, and different types of cancer, such as hepatocellular carcinoma. An increasing number of publicans related to cellular function of lncRNAs shows that lncRNAs are receiving more and more attention. It has been shown that the function of lncRNAs is associated with their unique subcellular localization spatial and temporal patterns $[8,2,11]$.

Currently, there are two methods to obtain the subcellular localization of lncRNA. The first method is to obtain the subcellular location of IncRNAs by biochemical experiments, which could obtain accurate and reliable information. However, a long-time consumption and high cost due to the biochemical experiments impedes the study progress. The second approach is to use bioinformatics technology to predict the subcellular location of lncRNA through computer-related techniques and algorithms, which has the advantage of saving time, financial and material resources, and the disadvantage of certain errors.

Some researchers have already explored the subcellular localization of non-coding RNAs [3, 7]. Zhang et al. constituted a database called RNALocate, to provide efficient information of RNA subsellular localization to help researchers determine the association between RNA and subcellular localization, and develop new predictor. The prediction of subcellular localization of lncRNAs in the way of machine learning have also been developed [14].

Most of the method use k-mer as the feature to train the machine learning model [9]. k-mer has biological significance. K-mers are subsequences of length $k$ within a biological sequence. A lncRNA sequence of length $L$ will have $L-k+1 \mathrm{k}$-mers and $4^{k}$ possible kmers. $4^{k}$ different k-mers have the ability to characterize each lncRNA sequence [10].

Zhen et al. developed a prediction model called lncLocator to predict subcellular localization of lncRNAs. They constructed a benchmark dataset by extracting and screening the sequence from RNALocate, utilizing CD-hit to avoid the bias, and used Supervised Over-Sampling Method (SOS) to alleviate the negative effects from the imbalanced database $[4,12]$. The final benchmark dataset had 612 entries: 301 sequences in Cytoplasm, 152 in Nucleus, 91 in Cytosol, 43 in Ribosome, and 25 in Exosome. They trained four classifiers, which are the combinations of 4-mer features, high-level abstraction features, support vector machine $(\mathrm{SVM})$, and random forest $(\mathrm{RF})$ respectively $[5,6]$. The final result 
was a combination of the four classifiers and could predict five subcellular localizations of lncRNAs, including cytoplasm, nucleus, cytosol, ribosome and exosome. The overall accuracy based on their benchmark dataset is 0.59 [4].

Zhen et al. developed a prediction model called iLoc-lncRNA. They constructed a benchmark dataset by extracting and screening the sequence from RNALocate, and utilizing CD-hit to avoid the bias [15]. The final benchmark dataset had 426 entries: 301 sequences in Cytoplasm, 156 in Nucleus, 91 in Cytosol, 43 in Ribosome, and 30 in Exosome [15]. The final result was a predictor trained by SVM with the feature 8-mer, and it could predict four subcellular localizations of lncRNAs, including cytoplasm, nucleus, ribosome and exosome. The overall accuracy based on their benchmark dataset is 0.87 . However, both of them constructed the benchmark database by the RNALocate version 1.0. RNALocate have updated to the version 2.0 with many changes.

Both of the two predictors, IncLocator and iLoc-lncRNA, did not release the source code to the public. So, we could not evaluate the performance of the algorithm, but we could evaluate the performance of the trained model on the new dataset.

In this study, we are going to contrstruct a new dataset with the RNALocate v2.0 to evaluate the performance of the existing and achievable predictor lncLocator and iLoc-lncRNA.

\section{METHOD}

\subsection{Data Collection}

We collected the detailed information about lncRNA subcellular localizations from a web-accessible database, RNALocate (http://www.rna-society.org/rnalo cate/). The current version of RNALocate is V2.0, which was updated latest in November 2019. It documents more than 190,000 RNA-associated sub-cellular localization entries with experimental and predicted evidence, involving more than 105,000 RNAs with 44 sub-cellular localizations in 65 species, mainly including Homo sapiens, Mus musculus, and Saccharomyces cerevisiae, etc [14].

Our goal is to extract the lncRNA sequences from Homo sapiens located in the four subcellular locations with most lncRNAs.

The steps of data collection are described below:

1) Download RNA entries and extract lncRNA entries from RNALocate.

2) Screen out the entries without website link to NCBI or Ensembl database.

3) Keep entries in four subcellular locations: nucleus, cy- tosol, ribosome, and exosome.

4) For the current study, screen off the entries of multilocational lncRNA in the database, and retain the lncRNA samples located in only one subcellular location.

5) To extract the sequence, utilize Biopython for the lncRNA entries from NCBI database, and Ensemble Rest for the lncRNA entries from Ensembl database. Obtain the transcript ID from the NCBI webpage and Ensemble Rest. Drop the entry with no transcript information provided.

6) Extract the sequence in FASTA format based on the transcript ID.

7) Use CD-HIT to remove redundant sequences at an identity cut-off of $80 \%$ [12].
8) The final dataset has total 7055 entries: 30 sequences in cytoplasm, 40 in ribosome, 195 in nucleus, and 6790 in exosome.

\subsection{Predicted Data}

Both of the two predictors, IncLocator and iLoc-lncRNA, have their own existing servers available online at the address: www.csbio.sjtu.edu.cn/bioinf/lncLocator and http://lingroup.cn/server/iLoc-LncRNA, respectively [4] [15]. We submitted our dataset in FASTA to the online server, and fetch the predicted results by the web scrawler method using BeautifulSoup and python.

\subsection{Evaluation}

We use overall accuracy, precision, recall, f1-score, and confusion matrix as the evaluation criteria to evaluate the performance of the lncLocator and iLoc-lncRNA predictor on the new dataset.

$$
\begin{array}{r}
\text { Accuracy }=\frac{T P+T N}{T P+T N+F P+F N} \\
\text { Precision }^{(i)}=\frac{T P^{(i)}}{T P^{(i)}+F P^{(i)}} \\
\operatorname{Recall}^{(i)}=\frac{T P^{(i)}}{T P^{(i)}+F N^{(i)}} \\
F_{1}^{(i)}=2 \cdot \text { Precision }^{(i)} \cdot \operatorname{Recall}^{(i)} \\
\text { Precision }^{(i)}+\operatorname{Recall}^{(i)}
\end{array}
$$

Where $T P$ is true positive, $T N$ is true negative, $F P$ is false positive, $F N$ is true negative, and $i$ represents the class.

\section{RESULTS AND DISCUSSIONS 3.1 Experiment Results}

The overall accuracy of IncLocator is $3.91 \%$, and the overall accuracy of iLoc-lncRNA is $10.57 \%$. The results obtained by the lncLocator and iLoc-lncRNA predictor directly for overall accuracy, precision, recall and F1 are listed in Table 1 and Table 2.

Table 1. The results obtained by the IncLocator

\begin{tabular}{lllll}
\hline & \multicolumn{3}{l}{ lncLocator } & \\
\cline { 2 - 5 } & Precision & Recall & $\mathrm{F}_{1}$ & Accuracy \\
& $(\%)$ & $(\%)$ & $(\%)$ & $(\%)$ \\
\hline Cytosol & 00.49 & 96.67 & 00.98 & 03.91 \\
Exosome & 96.33 & 03.09 & 05.99 & \\
Nucleus & 04.16 & 15.90 & 06.60 & \\
Ribosome & 03.00 & 15.00 & 05.00 & \\
\hline Macro avg & 26.00 & 32.66 & 04.64 & \\
\hline Weighted avg & 92.85 & 03.91 & 05.98 & \\
\hline
\end{tabular}

From the table above, we recognized that the overall performance and the performance of each class were susceptible to the new dataset. This implies that both of the models do not hold the current database in the previous accuracy. 
Table 2. The results obtained by the iLoc-IncRNA

\begin{tabular}{lllll}
\hline & \multicolumn{3}{l}{ iLoc-lncRNA } & \\
\cline { 2 - 5 } & Precision & Recall & $\mathrm{F}_{1}$ & Accuracy \\
& $(\%)$ & $(\%)$ & $(\%)$ & $(\%)$ \\
\hline Cytosol & 00.54 & 70.00 & 01.06 & 10.57 \\
Exosome & 98.20 & 09.66 & 17.59 & \\
Nucleus & 03.23 & 29.23 & 05.82 & \\
Ribosome & 01.70 & 30.00 & 03.23 & \\
\hline Macro avg & 25.92 & 34.72 & 06.93 & \\
\hline Weighted avg & 94.62 & 10.57 & 17.11 & \\
\hline
\end{tabular}

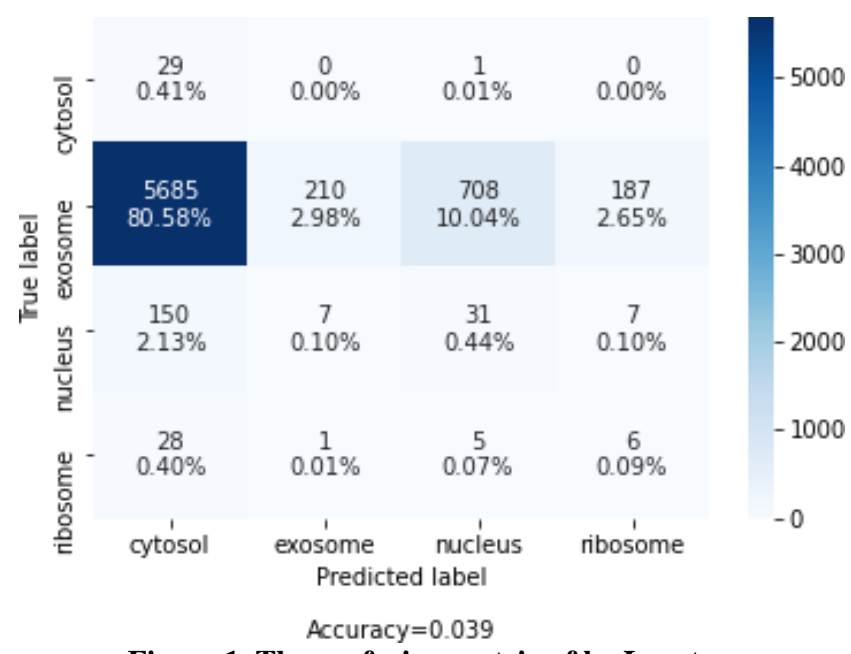

Figure 1. The confusion matrix of IncLocator.

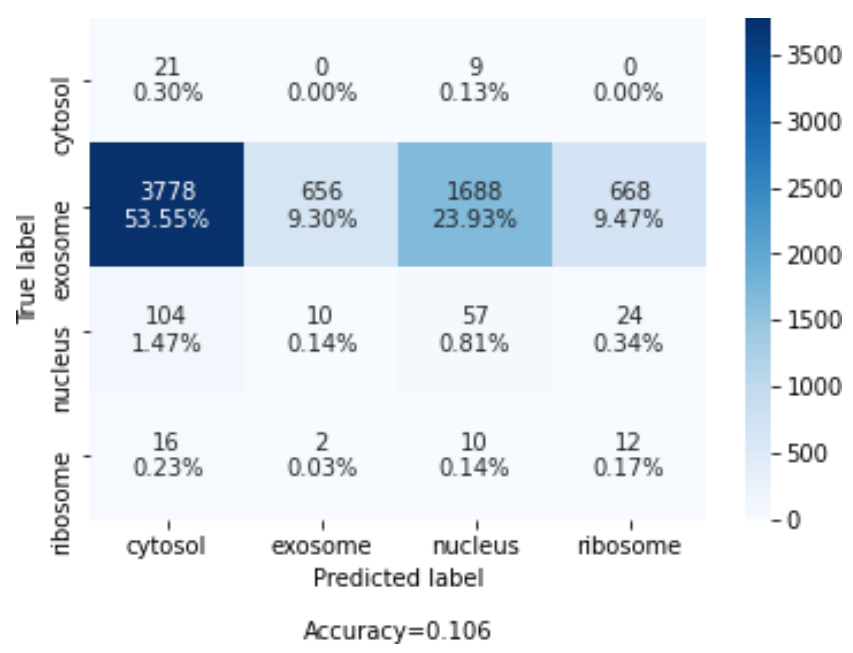

Figure 2. The confusion matrix of iLoc-IncRNA.

Especially, there is a large difference between the macro average of precision and the weighted average of precision, where the macro avg of precision is $26.00 \%$ and weighted avg of precision is $92.85 \%$ achieved by lncLocator, and the macro avg of precision is $25.92 \%$ and weighted avg of precision is $94.62 \%$ achieved by iLoclncRNA. The low macro avg of precision and high weighted avg of precision implied that each class was highly affected by the imbalanced dataset. The problem is probably because current dataset is extremely imbalanced. Because the precision and $F_{1}$ score of the class Exosome is extremely higher than that of others, and the recall of the class Exosome is extremely lower than that of others. The difference between the two datasets would be discussed later.

Confusion matrix is a table describing the performance of a model directly. The effect of imbalanced dataset could be clearly displayed by the confusion matrix. Figure 1 and Figure 2 shows the confusion matrix of each predictor respectively.

We see that most of them are predicted as Cytosol or Cytoplasm, which is the largest class in the datasets they used to train the two existing predictors.

\subsection{Discussions}

The results show that the performance of both predictors on their own benchmark database is better than the performance on the new database extracted in a similar way to the original one. This is due to the large difference between the two versions of the database, RNALocate v1.0 and RNALocate v2.0. The count number of each class in the three datasets is listed in the Table 3.

Table 3. Benchmark dataset

\begin{tabular}{llll}
\hline & lncLocator & iLoc-lncRNA & Present \\
\cline { 2 - 4 } Cytoplasm & 301 & 426 & 30 \\
Exosome & 25 & 30 & 6790 \\
Nucleus & 152 & 156 & 195 \\
Ribosome & 42 & 43 & 40 \\
\hline
\end{tabular}

It is obvious that two classes, Cytoplasm and Exosome, have large difference between the two versions of the database, especially the class Exosome. Exosome becomes the extreme majority class, and Cytoplasm becomes the minority class in the current database. More importantly, Exosome accounts for about $96 \%$ of the current database. While the majority class in the benchmark database of IncLocator and iLoc-lncRNA, Cytoplasm, accounts for 58\% and $65 \%$, respectively. The large discrepancy between the distribution of the two versions of the database results in the decreasing performance. The difference between the two versions of the database may because large number of biochemical experiments are always in progress. As a result, new genome entry is determined and characterized, while some old genome entries are corrected.

Besides the discrepancy in the proportion of the majority classes between the former database and the new one, $96 \%$ is still a large number to the common prediction model, such as SVM and RF. To alleviate this effect, one of the methods is to make the model more complex. From the evaluation of lncLocator and iLoc-lncRNA, we know that the predictor based on 8-mer feature performs better than the predictor with 4-mer feature. Hence, we could consider expanding the dimension of $\mathrm{k}$-mer feature to make the model complicated while we also have to avoid over-fitting. Besides the $\mathrm{k}$-mer feature, more kinds of feature with biological significance are expected to be extracted from the sequence data to improve the performance of the future model.

In addition, the common machine learning method used in predicting subcellular localization, such as SVM and RF, may not be able to deal with the extreme genome dataset in the future. We will pay attention to utilizing deep learning and neural network to build the prediction model as the future plan. Also, it would be 
better to update and train the existing predictor again with the new released database.

\section{CONCLUSION}

In this paper, we constructed a dataset including four classes based on the RNALocate v2.0 instead of RNALocate v1.0 to evaluate the performance of two existing predictors, lncLocator and iLoclncRNA. However, the extremely imbalanced dataset based on the constantly updated database and the lack of the feature extracted from the sequence data may be a problem in constructing a wellperformed model. More complex feature and more kinds of feature with biological significance are in demand to make models of higher complexity. The introduction of deep learning and neural network, and new designed algorithm may be expected. Also, to make a clear comparison between different models predicting different classes based on various databases, standard steps and criterion are in high demand.

\section{REFERENCES}

[1] Birney, E., Stamatoyannopoulos, J., Dutta, A. et al. Identification and analysis of functional elements in $1 \%$ of the human genome by the ENCODE pilot project. Nature 447, 799-816 (2007). https://doi.org/10.1038/nature05874.

[2] Broude N. E. (2011). Analysis of RNA localization and metabolism in single live bacterial cells: achievements and challenges. Molecular microbiology, 80(5), 1137-1147. https://doi.org/10.1111/j.1365-2958.2011.07652.x.

[3] Cai, Y. D., Liu, X. J., Xu, X. B., \& Chou, K. C. (2002). Support vector machines for prediction of protein subcellular location by incorporating quasi-sequence-order effect. Journal of cellular biochemistry, 84(2), 343-348. https://doi.org/10.1002/jcb.10030.

[4] Cao, Z., Pan, X., Yang, Y., Huang, Y., \& Shen, H. B. (2018). The lncLocator: a subcellular localization predictor for long non-coding RNAs based on a stacked ensemble classifier. Bioinformatics (Oxford, England), 34(13), 2185-2194. https://doi.org/10.1093/bioinformatics/bty085.

[5] Chen, W., Lei, T. Y., Jin, D. C., Lin, H., \& Chou, K. C. (2014). PseKNC: a flexible web server for generating pseudo K-tuple nucleotide composition. Analytical biochemistry, 456, 53-60. https://doi.org/10.1016/j.ab.2014.04.001.

[6] Chih-Chung Chang and Chih-Jen Lin. 2011. LIBSVM: A library for support vector machines. ACM Trans. Intell. Syst. Technol. 2, 3, Article 27 (April 2011), 27 pages. DOI:https://doi.org/10.1145/1961189.1961199.
[7] Chou, K. C., \& Shen, H. B. (2007). Recent progress in protein subcellular location prediction. Analytical biochemistry, 370(1), 1-16. https://doi.org/10.1016/j.ab.2007.07.006.

[8] DiStefano J. K. (2018). The Emerging Role of Long Noncoding RNAs in Human Disease. Methods in molecular biology (Clifton, N.J.), 1706, 91-110. https://doi.org/10.1007/978-1-4939-7471-9_6.

[9] Feng, P., Yang, H., Ding, H., Lin, H., Chen, W., \& Chou, K. C. (2019). iDNA6mA-PseKNC: Identifying DNA Nmethyladenosine sites by incorporating nucleotide physicochemical properties into PseKNC. Genomics, 111(1), 96-102. https://doi.org/10.1016/j.ygeno.2018.01.005.

[10] Ghandi, M., Mohammad-Noori, M., \& Beer, M. A. (2014). Robust k-mer frequency estimation using gapped k-mers. Journal of mathematical biology, 69(2), 469-500. https://doi.org/10.1007/s00285-013-0705-3.

[11] Gupta, R. A., Shah, N., Wang, K. C., Kim, J., Horlings, H. M., Wong, D. J., Tsai, M. C., Hung, T., Argani, P., Rinn, J. L., Wang, Y., Brzoska, P., Kong, B., Li, R., West, R. B., van de Vijver, M. J., Sukumar, S., \& Chang, H. Y. (2010). Long non-coding RNA HOTAIR reprograms chromatin state to promote cancer metastasis. Nature, 464(7291), 1071-1076. https://doi.org/10.1038/nature08975..

[12] Li, W., \& Godzik, A. (2006). Cd-hit: a fast program for clustering and comparing large sets of protein or nucleotide sequences. Bioinformatics (Oxford, England), 22(13), 16581659. https://doi.org/10.1093/bioinformatics/btl158.

[13] Spizzo, R., Almeida, M. I., Colombatti, A., \& Calin, G. A. (2012). Long non-coding RNAs and cancer: a new frontier of translational research?. Oncogene, 31(43), 4577-4587. https://doi.org/10.1038/onc.2011.621.

[14] Zhang, T., Tan, P., Wang, L., Jin, N., Li, Y., Zhang, L., Yang, H., Hu, Z., Zhang, L., Hu, C., Li, C., Qian, K., Zhang, C., Huang, Y., Li, K., Lin, H., \& Wang, D. (2017). RNALocate: a resource for RNA subcellular localizations. Nucleic acids research, 45(D1), D135-D138. https://doi.org/10.1093/nar/gkw728.

[15] Zhen-Dong Su, Yan Huang, Zhao-Yue Zhang, Ya-Wei Zhao, Dong Wang, Wei Chen, Kuo-Chen Chou, Hao Lin, iLoclncRNA: predict the subcellular location of lncRNAs by incorporating octamer composition into general PseKNC, Bioinformatics, Volume 34, Issue 24, 15 December 2018, Pages 4196-4204, https://doi.org/10.1093/bioinformatics/bty508. 